\title{
Regular polygons, Morgan-Voyce polynomials, and Chebyshev polynomials
}

\author{
Jorma K. Merikoski \\ Faculty of Information Technology and Communication Sciences, Tampere University \\ FI-33014 Tampere, Finland \\ e-mail: jorma.merikoski@tuni.fi
}

Received: 14 July 2020

Revised: 9 April 2021

Accepted: 18 April 2021

\begin{abstract}
We say that a monic polynomial with integer coefficients is a polygomial if its each zero is obtained by squaring the edge or a diagonal of a regular $n$-gon with unit circumradius. We find connections of certain polygomials with Morgan-Voyce polynomials and further with Chebyshev polynomials of second kind.
\end{abstract}

Keywords: Regular polygons, Morgan-Voyce polynomials, Chebyshev polynomials, Vieta polynomials.

2020 Mathematics Subject Classification: 11B83, 51M20.

\section{Introduction}

We call the edge and diagonals of a polygon by a common name chord. Let $G_{n}$ be a regular $n$-gon with unit circumradius. Its chords (their lengths) are

$$
e_{n k}=2 \sin \frac{k \pi}{n}, \quad k=1, \ldots,\left\lfloor\frac{n}{2}\right\rfloor .
$$

We say that a monic polynomial with integer coefficients is a polygomial if its all zeros are squared chords (not necessarily squares of all chords) of some $G_{n}$.

This paper is a sequel to Mustonen et al. [7, Section 2] on the polygomials

$$
A_{m}(x)=\sum_{k=0}^{m}(-1)^{m-k}\left(\begin{array}{c}
m+k+1 \\
2 k+1
\end{array}\right) x^{k}=\prod_{k=1}^{m}\left[x-4 \sin ^{2} \frac{k \pi}{2(m+1)}\right]
$$

(for the second equation, see [7, Theorem 1]) and 


$$
\tilde{A}_{m}(x)=x^{m}+\sum_{k=0}^{m-1}(-1)^{m-k} \frac{2 m+1}{m-k}\left(\begin{array}{c}
m+k \\
2 k+1
\end{array}\right) x^{k}=\prod_{k=1}^{m}\left[x-4 \sin ^{2} \frac{k \pi}{2 m+1}\right]
$$

(for the second equation, see [7, Theorem 2]). Define that the "empty sum" is zero and the "empty product" one; then $A_{0}(x)=\tilde{A}_{0}(x)=1$. In [7], $B_{m}=\tilde{A}_{m}$ and $\tilde{A}_{m}$ has another meaning.

Example 1.1. In particular,

$$
\begin{array}{r}
A_{1}(x)=x-2, \quad A_{2}(x)=x^{2}-4 x+3, \quad A_{3}(x)=x^{3}-6 x^{2}+10 x-4, \\
A_{4}(x)=x^{4}-8 x^{3}+21 x^{2}-20 x+5, \\
\tilde{A}_{1}(x)=x-3, \quad \tilde{A}_{2}(x)=x^{2}-5 x+5, \quad \tilde{A}_{3}(x)=x^{3}-7 x^{2}+14 x-7, \\
\tilde{A}_{4}(x)=x^{4}-9 x^{3}+27 x^{2}-30 x+9 .
\end{array}
$$

The sequence $\left(A_{m}\right)$ satisfies [7, Equation (6)] the recursion

$$
A_{0}(x)=1, \quad A_{1}(x)=x-2, \quad A_{m+1}(x)=(x-2) A_{m}(x)-A_{m-1}(x),
$$

and $\left(\tilde{A}_{m}\right)$ satisfies [7, Equation (11)]

$$
\tilde{A}_{0}(x)=1, \quad \tilde{A}_{1}(x)=x-3, \quad \tilde{A}_{m+1}(x)=(x-3) A_{m}(x)-A_{m-1}(x) .
$$

We show that

$$
\tilde{A}_{0}(x)=1, \quad \tilde{A}_{1}(x)=x-3, \quad \tilde{A}_{m+1}(x)=(x-2) \tilde{A}_{m}(x)-\tilde{A}_{m-1}(x) .
$$

Thus $\left(\tilde{A}_{m}\right)$ follows the same recursion formula as $\left(A_{m}\right)$.

For all $k \geq 2$,

$$
\begin{aligned}
\tilde{A}_{k}(x) & \stackrel{(3)}{=}(x-3) A_{k-1}(x)-A_{k-2}(x) \\
& \stackrel{(2)}{=}(x-3) A_{k-1}(x)+A_{k}(x)-(x-2) A_{k-1}(x)=A_{k}(x)-A_{k-1}(x) .
\end{aligned}
$$

Therefore

$$
\begin{aligned}
& \tilde{A}_{m+1}(x)-(x-2) \tilde{A}_{m}(x)+\tilde{A}_{m-1}(x) \\
& \stackrel{(5)}{=} A_{m+1}(x)-A_{m}(x)-(x-2)\left(A_{m}(x)-A_{m-1}(x)\right)+A_{m-1}(x)-A_{m-2}(x) \\
& =A_{m+1}(x)-(x-2) A_{m}(x)+A_{m-1}(x)-\left[A_{m}(x)-(x-2) A_{m-1}(x)+A_{m-2}(x)\right] \\
& \stackrel{(2)}{=} 0-0=0,
\end{aligned}
$$

verifying the claim.

We are interested in connections of $A_{m}$ and $\tilde{A}_{m}$ with well-known polynomials. We introduce in Sections 3 and 4 the Morgan-Voyce polynomials $b_{m}$ and $B_{m}$, and their generalizations $B_{m}^{(r)}$. We see that $A_{m}$ and $\tilde{A}_{m}$ are connected with $B_{m}$ and $B_{m}^{(2)}$, respectively. We also find a polygomial $a_{m}$ connected with $b_{m}$. In Section 5 , recalling how $b_{m}, B_{m}$, and $B_{m}^{(2)}$ reduce to the Chebyshev polynomials of second kind, we reduce also $a_{m}, A_{m}$, and $\tilde{A}_{m}$ to them. The motivation of $b_{m}$ and $B_{m}$ rises from a problem on a ladder network of resistances. We see in Section 6 that also $a_{m}$ and $A_{m}$ apply to this problem. Finally, we complete this paper with conclusions and remarks in Section 7. 


\section{Background}

Let me first describe the background of this paper. Neeme Vaino, an amateur mathematician from Estonia, introduced [11] his "regular polynomials"

$$
R_{n}(x)=\sum_{k=0}^{\left\lfloor\frac{n}{2}\right\rfloor} r_{n k} x^{n-2 k},
$$

whose coefficients are obtained from the OEIS [8] sequence A132460. Actually [5, Equation (1.5)] $R_{n}$ is the Vieta-Lucas polynomial

$$
v_{n}(x)=\sum_{k=0}^{\left\lfloor\frac{n}{2}\right\rfloor}(-1)^{k} \frac{n}{n-k}\left(\begin{array}{c}
n-k \\
k
\end{array}\right) x^{n-2 k} .
$$

Example 2.1. In particular,

$$
\begin{array}{r}
v_{1}(x)=x, \quad v_{2}(x)=x^{2}-2, \quad v_{3}(x)=x^{3}-3 x, \quad v_{4}(x)=x^{4}-4 x^{2}+2, \\
v_{5}(x)=x^{5}-5 x^{3}+5 x, \quad v_{6}(x)=x^{6}-6 x^{4}+9 x^{2}-2 .
\end{array}
$$

The polygomial $\tilde{A}_{m}$ relates to $v_{2 m+1}$ via

$$
v_{2 m+1}(x)=x \tilde{A}_{m}\left(x^{2}\right),
$$

cf. [5, Theorem 3(b)].

Example 2.2. In particular,

$$
x \tilde{A}_{2}\left(x^{2}\right)=x\left(x^{4}-5 x^{2}+5\right)=x^{5}-5 x^{3}+5 x=v_{5}(x) .
$$

The Chebyshev polynomials of first kind are defined by

$$
T_{0}(x)=1, \quad T_{1}(x)=x, \quad T_{n+1}(x)=2 x T_{n}(x)-T_{n-1}(x) .
$$

Example 2.3. In particular,

$$
\begin{array}{r}
T_{1}(x)=x, \quad T_{2}(x)=2 x^{2}-1, \quad T_{3}(x)=4 x^{3}-3 x, \quad T_{4}(x)=8 x^{4}-8 x^{2}+1, \\
T_{5}(x)=16 x^{5}-20 x^{3}+5 x, \quad T_{6}(x)=32 x^{6}-48 x^{4}+18 x^{2}-1 .
\end{array}
$$

The polynomial $v_{n}$ relates to $T_{n}\left[5\right.$, Equation (9.4)] via $v_{n}(x)=2 T_{n}\left(\frac{x}{2}\right)$.

Example 2.4. In particular,

$$
2 T_{4}\left(\frac{x}{2}\right)=2 \cdot\left[8\left(\frac{x}{2}\right)^{4}-8\left(\frac{x}{2}\right)^{2}+1\right]=x^{4}-4 x^{2}+2=v_{4}(x) .
$$

The Vieta-Fibonacci polynomials are defined by

$$
V_{n}(x)=\sum_{k=0}^{\left\lfloor\frac{n}{2}\right\rfloor}(-1)^{k}\left(\begin{array}{c}
n-k \\
k
\end{array}\right) x^{n-2 k},
$$

cf. [5, Equation (1.3)]. Here $V_{n}$ denotes the same as $V_{n+1}$ in [5], in order to make its degree equal to $n$. 
Example 2.5. In particular,

$$
\begin{array}{r}
V_{1}(x)=x, \quad V_{2}(x)=x^{2}-1, \quad V_{3}(x)=x^{3}-2 x, \quad V_{4}(x)=x^{4}-3 x^{2}+1, \\
V_{5}(x)=x^{5}-4 x^{3}+3 x, \quad V_{6}(x)=x^{6}-5 x^{4}+6 x^{2}-1 .
\end{array}
$$

The polygomial $A_{m}$ relates to $V_{2 m+1}$ via $V_{2 m+1}(x)=x A_{m}\left(x^{2}\right)$, cf. [5, Theorem 2(a)].

Example 2.6. In particular,

$$
x A_{2}\left(x^{2}\right)=x\left(x^{4}-4 x^{2}+3\right)=x^{5}-4 x^{3}+3 x=V_{5}(x) .
$$

The Chebyshev polynomials of second kind are defined by

$$
U_{0}(x)=1, \quad U_{1}(x)=2 x, \quad U_{n+1}(x)=2 x U_{n}(x)-U_{n-1}(x) .
$$

Example 2.7. In particular,

$$
\begin{array}{r}
U_{1}(x)=2 x, \quad U_{2}(x)=4 x^{2}-1, \quad U_{3}(x)=8 x^{3}-4 x, \quad U_{4}(x)=16 x^{4}-12 x^{2}+1, \\
U_{5}(x)=32 x^{5}-32 x^{3}+6 x, \quad U_{6}(x)=64 x^{6}-80 x^{4}+24 x^{2}-1 .
\end{array}
$$

The polynomial $V_{n}$ relates to $U_{n}$ via $V_{n}(x)=U_{n}\left(\frac{x}{2}\right)$, cf. [5, Equation (9.3)]. Actually $V_{n}(x)$ should read $V_{n+1}(x)$ in this reference, in order to make (9.3) compatible with (1.3).

Example 2.8. In particular,

$$
U_{4}\left(\frac{x}{2}\right)=16\left(\frac{x}{2}\right)^{4}-12\left(\frac{x}{2}\right)^{2}+1=x^{4}-3 x^{2}+1=V_{4}(x) .
$$

To summarize, $A_{m}$ relates to $V_{2 m+1}$ and further to $U_{2 m+1}$, and $\tilde{A}_{m}$ relates to $v_{2 m+1}$ and further to $T_{2 m+1}$. But we will see that $A_{m}$ and $\tilde{A}_{m}$ have also more direct relations to well-known polynomials.

\section{Morgan-Voyce polynomials}

Changing in $A_{m}$ all minus signs into plus, we define

$$
B_{m}(x)=(-1)^{m} A_{m}(-x) .
$$

This is one of the two Morgan-Voyce polynomials $b_{m}$ and $B_{m}$, usually defined by the recursion pair

$$
b_{0}(x)=B_{0}(x)=1, \quad b_{m+1}(x)=x B_{m}(x)+b_{m}(x), \quad B_{m+1}(x)=(x+1) B_{m}(x)+b_{m}(x) .
$$

Example 3.1. In particular,

$$
\begin{array}{r}
b_{1}(x)=x+1, \quad b_{2}(x)=x^{2}+3 x+1, \quad b_{3}(x)=x^{3}+5 x^{2}+6 x+1, \\
b_{4}(x)=x^{4}+7 x^{3}+15 x^{2}+10 x+1, \\
B_{1}(x)=x+2, \quad B_{2}(x)=x^{2}+4 x+3, \quad B_{3}(x)=x^{3}+6 x^{2}+10 x+4, \\
B_{4}(x)=x^{4}+8 x^{3}+21 x^{2}+20 x+5 .
\end{array}
$$


By (6) and (2),

$$
B_{0}(x)=1, \quad B_{1}(x)=x+2, \quad B_{m+1}(x)=(x+2) B_{m}(x)-B_{m-1}(x) .
$$

This recursion is well-known $[9$, p. 73] as a consequence of (7). Likewise,

$$
b_{0}(x)=1, \quad b_{1}(x)=x+1, \quad b_{m+1}(x)=(x+2) b_{m}(x)-b_{m-1}(x) .
$$

By (6) and (1),

$$
B_{m}(x)=\sum_{k=0}^{m}\left(\begin{array}{c}
m+k+1 \\
2 k+1
\end{array}\right) x^{k}=\prod_{k=1}^{m}\left[x+4 \sin ^{2} \frac{k \pi}{2(m+1)}\right] .
$$

Regarding zeros, this is well-known [9, Equation (39)] (the first of the two equations with this number) and [10, Section 6]. Similarly, by [9, Equation (40)] and [10, Section 6] (containing a typo),

$$
b_{m}(x)=\sum_{k=0}^{m}\left(\begin{array}{c}
m+k \\
2 k
\end{array}\right) x^{k}=\prod_{k=1}^{m}\left[x+4 \sin ^{2} \frac{(2 k-1) \pi}{2(2 m+1)}\right] .
$$

\section{Counterparts of $\tilde{A}_{m}$ and $b_{m}$}

Equation (6) connects $A_{m}$ and $B_{m}$ but does not connect $\tilde{A}_{m}$ and $b_{m}$. Instead, $b_{m}$ is connected with

$$
\begin{aligned}
a_{m}(x)=(-1)^{m} b_{m}(-x) & \stackrel{(8)}{=} \prod_{k=1}^{m}(-1)\left[-x+4 \sin ^{2} \frac{(2 k-1) \pi}{2(2 m+1)}\right] \\
& =\prod_{k=1}^{m}\left[x-4 \sin ^{2} \frac{(2 k-1) \pi}{2(2 m+1)}\right] .
\end{aligned}
$$

This is a polygomial, since its zeros are $e_{4 m+2,1}^{2}, e_{4 m+2,3}^{2}, \ldots, e_{4 m+2,2 m-1}^{2}$.

Example 4.1. In particular,

$$
\begin{array}{r}
a_{1}(x)=x-1, \quad a_{2}(x)=x^{2}-3 x+1, \quad a_{3}(x)=x^{3}-5 x^{2}+6 x-1, \\
a_{4}(x)=x^{4}-7 x^{3}+15 x^{2}-10 x+1 .
\end{array}
$$

The Morgan-Voyce polynomials have been widely generalized, see [4] and its references. André-Jeannin [1] generalizes them by the recursion

$$
B_{0}^{(r)}(x)=1, \quad B_{1}^{(r)}(x)=x+r+1, \quad B_{m+1}^{(r)}(x)=(x+2) B_{m}^{(r)}(x)-B_{m-1}^{(r)}(x),
$$

where $r$ is a given real number. In particular,

$$
B_{m}^{(0)}=b_{m}, \quad B_{m}^{(1)}=B_{m}
$$

and the polynomials

$$
\tilde{B}_{m}=B_{m}^{(2)}
$$

satisfy the recursion

$$
\tilde{B}_{0}(x)=1, \quad \tilde{B}_{1}(x)=x+3, \quad \tilde{B}_{m+1}(x)=(x+2) \tilde{B}_{m}(x)-\tilde{B}_{m-1}(x) .
$$


By (4) and (10), it is easy to see that

$$
\tilde{B}_{m}(x)=(-1)^{m} \tilde{A}_{m}(-x) .
$$

Example 4.2. In particular,

$$
\begin{array}{r}
\tilde{B}_{1}(x)=x+3, \quad \tilde{B}_{2}(x)=x^{2}+5 x+5, \quad \tilde{B}_{3}(x)=x^{3}+7 x^{2}+14 x+7 \\
\tilde{B}_{4}(x)=x^{4}+9 x^{3}+27 x^{2}+30 x+9 .
\end{array}
$$

\section{Chebyshev polynomials of second kind}

It can be shown [4, Equations (4.2-4)] that

$$
\begin{aligned}
B_{m}(x) & =U_{m}\left(\frac{x+2}{2}\right), \\
b_{m}(x) & =U_{m}\left(\frac{x+2}{2}\right)-U_{m-1}\left(\frac{x+2}{2}\right), \\
\tilde{B}_{m}(x) & =U_{m}\left(\frac{x+2}{2}\right)+U_{m-1}\left(\frac{x+2}{2}\right) .
\end{aligned}
$$

Example 5.1. In particular,

$$
\begin{gathered}
U_{1}\left(\frac{x+2}{2}\right)=2 \frac{x+2}{2}=x+2=B_{1}(x), \\
U_{2}\left(\frac{x+2}{2}\right)=4\left(\frac{x+2}{2}\right)^{2}-1=x^{2}+4 x+3=B_{2}(x), \\
U_{2}\left(\frac{x+2}{2}\right)-U_{1}\left(\frac{x+2}{2}\right)=x^{2}+3 x+1=b_{2}(x), \\
U_{2}\left(\frac{x+2}{2}\right)+U_{1}\left(\frac{x+2}{2}\right)=x^{2}+5 x+5=\tilde{B}_{2}(x) .
\end{gathered}
$$

It is easy to see that

$$
U_{m}(-x)=(-1)^{m} U_{m}(x) .
$$

Now,

$$
\begin{aligned}
A_{m}(x) & \stackrel{(6)}{=}(-1)^{m} B_{m}(-x) \stackrel{(12)}{=}(-1)^{m} U_{m}\left(\frac{-x+2}{2}\right) \stackrel{(15)}{=}(-1)^{2 m} U_{m}\left(\frac{x-2}{2}\right)=U_{m}\left(\frac{x-2}{2}\right), \\
a_{m}(x) & \stackrel{(9)}{=}(-1)^{m} b_{m}(-x) \stackrel{(13)}{=}(-1)^{m}\left(U_{m}\left(\frac{-x+2}{2}\right)-U_{m-1}\left(\frac{-x+2}{2}\right)\right) \\
& \stackrel{(15)}{=}(-1)^{m}\left[(-1)^{m} U_{m}\left(\frac{x-2}{2}\right)-(-1)^{m-1} U_{m-1}\left(\frac{x-2}{2}\right)\right]=U_{m}\left(\frac{x-2}{2}\right)+U_{m-1}\left(\frac{x-2}{2}\right)
\end{aligned}
$$

and

$$
\begin{aligned}
\tilde{A}_{m}(x) & \stackrel{(11)}{=}(-1)^{m} \tilde{B}_{m}(-x) \stackrel{(14)}{=}(-1)^{m}\left(U_{m}\left(\frac{-x+2}{2}\right)+U_{m-1}\left(\frac{-x+2}{2}\right)\right) \\
& \stackrel{(15)}{=}(-1)^{m}\left[(-1)^{m} U_{m}\left(\frac{x-2}{2}\right)+(-1)^{m-1} U_{m-1}\left(\frac{x-2}{2}\right)\right]=U_{m}\left(\frac{x-2}{2}\right)-U_{m-1}\left(\frac{x-2}{2}\right) .
\end{aligned}
$$


Example 5.2. In particular,

$$
\begin{aligned}
U_{1}\left(\frac{x-2}{2}\right) & =2 \frac{x-2}{2}=x-2=A_{1}(x), \\
U_{2}\left(\frac{x-2}{2}\right) & =4\left(\frac{x-2}{2}\right)^{2}-1=x^{2}-4 x+3=A_{2}(x), \\
U_{2}\left(\frac{x-2}{2}\right)+U_{1}\left(\frac{x-2}{2}\right) & =x^{2}-3 x+1=a_{2}(x), \\
U_{2}\left(\frac{x-2}{2}\right)-U_{1}\left(\frac{x-2}{2}\right) & =x^{2}-5 x+5=\tilde{A}_{2}(x) .
\end{aligned}
$$

\section{Revisiting the ladder network}

We show that $a_{m}$ and $A_{m}$ apply to the same ladder network problem $[2,6]$ as $b_{m}$ and $B_{m}$. To begin, we present a recursion pair for $a_{m}$ and $A_{m}$. Since

$$
\begin{aligned}
a_{m+1}(x) & \stackrel{(9)}{=}(-1)^{m+1} b_{m+1}(-x) \stackrel{(7)}{=}(-1)^{m+1}\left[(-x) B_{m}(-x)+b_{m}(-x)\right] \\
& =x(-1)^{m} B_{m}(-x)-(-1)^{m} b_{m}(-x) \stackrel{(6),(9)}{=} x A_{m}(x)-a_{m}(x)
\end{aligned}
$$

and

$$
\begin{aligned}
A_{m+1}(x) & \stackrel{(6)}{=}(-1)^{m+1} B_{m+1}(-x) \stackrel{(7)}{=}(-1)^{m+1}\left[(-x+1) B_{m}(-x)+b_{m}(-x)\right] \\
& =(x-1)(-1)^{m} B_{m}(-x)-(-1)^{m} b_{m}(-x) \stackrel{(6),(9)}{=}(x-1) A_{m}(x)-a_{m}(x),
\end{aligned}
$$

we have

$$
a_{0}(x)=A_{0}(x)=1, \quad a_{m+1}(x)=x A_{m}(x)-a_{m}(x), \quad A_{m+1}(x)=(x-1) A_{m}(x)-a_{m}(x) .
$$

We use the figures and notations of Hoggatt and Bicknell [2, Section 1]. Instead of $x$, we let $-x$ denote the resistance of each component in the upper sidepiece of the ladder. It is reasonable to require that $-x>0$, i.e., $x<0$. However, it is not complete nonsense to accept also nonpositive resistances, because we may think that the voltage across these components can be increased externally. Anyway, whether or not to accept nonpositive resistances, it does not effect on the following calculations.

We proceed as in [2, p. 148] but write $R(x)=R$ and $Z_{m}(x)=Z_{n}$. Then

$$
\begin{gathered}
R(x)=Z_{m}(x)-x, \\
\frac{1}{Z_{m+1}(x)}=\frac{1}{Z_{m}(x)-x}+1=\frac{Z_{m}(x)-x+1}{Z_{m}(x)-x},
\end{gathered}
$$

and

$$
Z_{m+1}(x)=\frac{Z_{m}(x)-x}{Z_{m}(x)-x+1} .
$$

We show that

$$
Z_{m}(x)=\frac{a_{m}(x)}{A_{m}(x)}
$$

satisfies (17). Since 


$$
\begin{aligned}
Z_{m+1}(x) & \stackrel{(18)}{=} \frac{a_{m+1}(x)}{A_{m+1}(x)} \stackrel{(16)}{=} \frac{x A_{m}(x)-a_{m}(x)}{(x-1) A_{m}(x)-a_{m}(x)} \\
& =\frac{a_{m}(x)-x A_{m}(x)}{a_{m}(x)-(x-1) A_{m}(x)}=\frac{\frac{a_{m}(x)}{A_{m}(x)}-x}{\frac{a_{m}(x)}{A_{m}(x)}-x+1} \stackrel{(18)}{=} \frac{Z_{m}(x)-x}{Z_{m}(x)-x+1},
\end{aligned}
$$

the claim follows.

\section{Conclusions and remarks}

The polygomial $A_{m}$ is connected with $B_{m}$ via the equation (6). The Morgan-Voyce polynomial $b_{m}$ defines by (9) the polygomial $a_{m}$. The polygomial $\tilde{A}_{m}$ has the connection (11) with the generalized Morgan-Voyce polynomial $\tilde{B}_{m}=B_{m}^{(2)}$. Since these Morgan-Voyce polynomials reduce to Chebyshev polynomials of second kind via (12), (13), and (14), also the above-mentioned polygomials reduce to them.

More generally, we define

$$
A_{m}^{(r)}(x)=(-1)^{m} B^{(r)}(-x)
$$

In particular,

$$
A_{m}^{(0)}=a_{m}, \quad A_{m}^{(1)}=A_{m}, \quad A_{m}^{(2)}=\tilde{A}_{m} .
$$

If $r \in\{0,1,2\}$, then $A_{m}^{(r)}$ is a polygomial. Is it a polygomial also for some other appropriate values of $r$ ? To answer, we should find the zeros of $B_{m}^{(r)}$. I did not find them from the literature. According to Horadam [3, p. 348], André-Jeannin [1] has given them, but actually he [1, p. 231] considered only the cases $r=0,1,2$.

\section{Acknowledgements}

Neeme Vaino's "regular polynomials" (discussed in Section 2) motivated this paper. Pentti Haukkanen gave useful references. I thank both of them.

\section{References}

[1] André-Jeannin, R. (1994). A generalization of Morgan-Voyce polynomials. The Fibonacci Quarterly, 32, 228-231.

[2] Hoggatt, V. E., \& Bicknell, M. (1974). A primer for the Fibonacci numbers: Part XIV. The Fibonacci Quarterly, 12, 147-156.

[3] Horadam, A. F. (1996). Polynomials associated with generalized Morgan-Voyce polynomials. The Fibonacci Quarterly, 34, 342-348. 
[4] Horadam, A. F. (1997). A composite of Morgan-Voyce generalizations. The Fibonacci Quarterly, 35, 233-239.

[5] Horadam, A, F. (2002). Vieta polynomials. The Fibonacci Quarterly, 40, 223-232.

[6] Morgan-Voyce, A. M. (1959). Ladder networks analysis using Fibonacci numbers. IRE Transactions on Circuit Theory, 6, 321-322.

[7] Mustonen, S., Haukkanen, P., \& Merikoski, J. (2014). Some polynomials associated with regular polygons. Acta Universitatis Sapientiae, Mathematica, 6, 178-193.

[8] OEIS: The On-Line Encyclopedia of Integer Sequences. Available online at http:// oeis.org/.

[9] Swamy, M. N. S. (1966). Properties of the polynomials defined by Morgan-Voyce. The Fibonacci Quarterly, 4, 73-81.

[10] Swamy, M. N. S. (1968). Further properties of Morgan-Voyce polynomials. The Fibonacci Quarterly, 6, 167-175.

[11] Vaino, N. (2020). Private communication. 